\title{
Examination of L-Glutamic Acid Polymorphs by Solid-State Density Functional Theory and Terahertz Spectroscopy
}

Michael T. Ruggiero ${ }^{\dagger}$, Juraj Sibik ${ }^{\ddagger}$, J. Axel Zeitler ${ }^{\ddagger}$, and Timothy M. Korter ${ }^{\dagger *}$

${ }^{\dagger}$ Department of Chemistry, Syracuse University, 1-014 Center for Science and Technology, Syracuse, NY 13244-4100, United States of America

* Department of Chemical Engineering and Biotechnology, University of Cambridge, New Museums Site, Pembroke Street, Cambridge CB2 3RA, United Kingdom

\section{Supporting Information}

Table S1. Relative energetic values (kJ/mol, per molecule) for the two studied glutamic acid polymorphs.

\begin{tabular}{|c|c|c|c|c|c|c|}
\hline & \multicolumn{3}{|c|}{ PBE } & \multicolumn{3}{c|}{ PBE-D3 } \\
\hline & Electronic & $\begin{array}{c}\text { Molecular } \\
\text { Conformational }\end{array}$ & Cohesive & Electronic & $\begin{array}{c}\text { Molecular } \\
\text { Conformational }\end{array}$ & Cohesive \\
\hline$\alpha$-GLU & 0 & 0 & 0 & 0 & 0 & 0 \\
\hline$\beta$-GLU & +8.23 & +3.97 & +4.26 & +3.69 & +4.83 & -1.14 \\
\hline
\end{tabular}


Table S2. Calculated vibrational frequencies (THz) and mode descriptions for the two Lglutamic acid polymorphs.

\begin{tabular}{|c|c|c|c|}
\hline & $\alpha-G L U$ & & $\beta-\mathrm{GLU}$ \\
\hline Freq & Mode Description & Freq & Mode Description \\
\hline 1.923 & Rotation about $b$ & 1.229 & Rotation about $b$ \\
\hline 2.070 & Translation in $c$ & 1.958 & Rotation about $a$ \\
\hline 2.120 & Rotation about $b c$ & 2.050 & Rotation about $c$ \\
\hline 2.284 & Rotation about $a$ & 2.388 & Torsion of $\mathrm{CH}_{2}-\mathrm{CH}$ bond \\
\hline 2.507 & Rotation about $c$ & 2.504 & Torsional bending of $\mathrm{COO}^{-}$and backbone \\
\hline 2.532 & Rotation about $b$ & 2.723 & Torsion of $\mathrm{COOH}$ about the $\mathrm{C}-\mathrm{C}$ bond \\
\hline 2.613 & Rotation about $b$ & 2.816 & Torsional bending about the backbone \\
\hline 2.713 & Torsional bending of $\mathrm{COO}^{-}$and backbone & 2.923 & Torsion of $\mathrm{CH}_{2}-\mathrm{CH}$ bond \\
\hline 2.840 & Torsion of $\mathrm{COOH}$ about the $\mathrm{C}-\mathrm{C}$ bond & 3.075 & Torsion of $\mathrm{COO}^{-}$about the $\mathrm{C}-\mathrm{C}$ bond \\
\hline 2.988 & Torsion of $\mathrm{COO}^{-}$about the $\mathrm{C}-\mathrm{C}$ bond & 3.313 & Torsion about the $\mathrm{CH}_{2}-\mathrm{CH}_{2}$ bond \\
\hline 3.071 & Torsional bending about the backbone & 3.350 & $\begin{array}{l}\text { Torsion about the backbone with } \mathrm{COO}^{-} \\
\text {and } \mathrm{COOH} \text { rotation }\end{array}$ \\
\hline 3.160 & $\begin{array}{l}\text { Torsion of } \mathrm{COO}^{-} \text {and } \mathrm{COOH} \text { about the } \mathrm{C}- \\
\mathrm{C} \text { bonds }\end{array}$ & 3.428 & $\begin{array}{l}\text { Torsion about the backbone with } \mathrm{COO}^{-} \\
\text {and } \mathrm{COOH} \text { rotation }\end{array}$ \\
\hline 3.330 & $\begin{array}{l}\text { Torsion of } \mathrm{COO}^{-} \text {and } \mathrm{COOH} \text { about the } \mathrm{C}- \\
\mathrm{C} \text { bonds }\end{array}$ & 3.536 & Torsion about the $\mathrm{CH}_{2}-\mathrm{CH}_{2}$ bond \\
\hline 3.600 & Torsion about the $\mathrm{CH}_{2}-\mathrm{CH}_{2}$ bond & 3.584 & Torsion of $\mathrm{COOH}$ about the $\mathrm{C}-\mathrm{C}$ bond \\
\hline 3.820 & $\begin{array}{c}\text { Torsion about the backbone with } \mathrm{COO}^{-} \\
\text {and } \mathrm{COOH} \text { rotation }\end{array}$ & 3.706 & Torsion about the backbone \\
\hline
\end{tabular}




\section{a-GLU Experimental X-ray Crystallographic Data (CIF)}

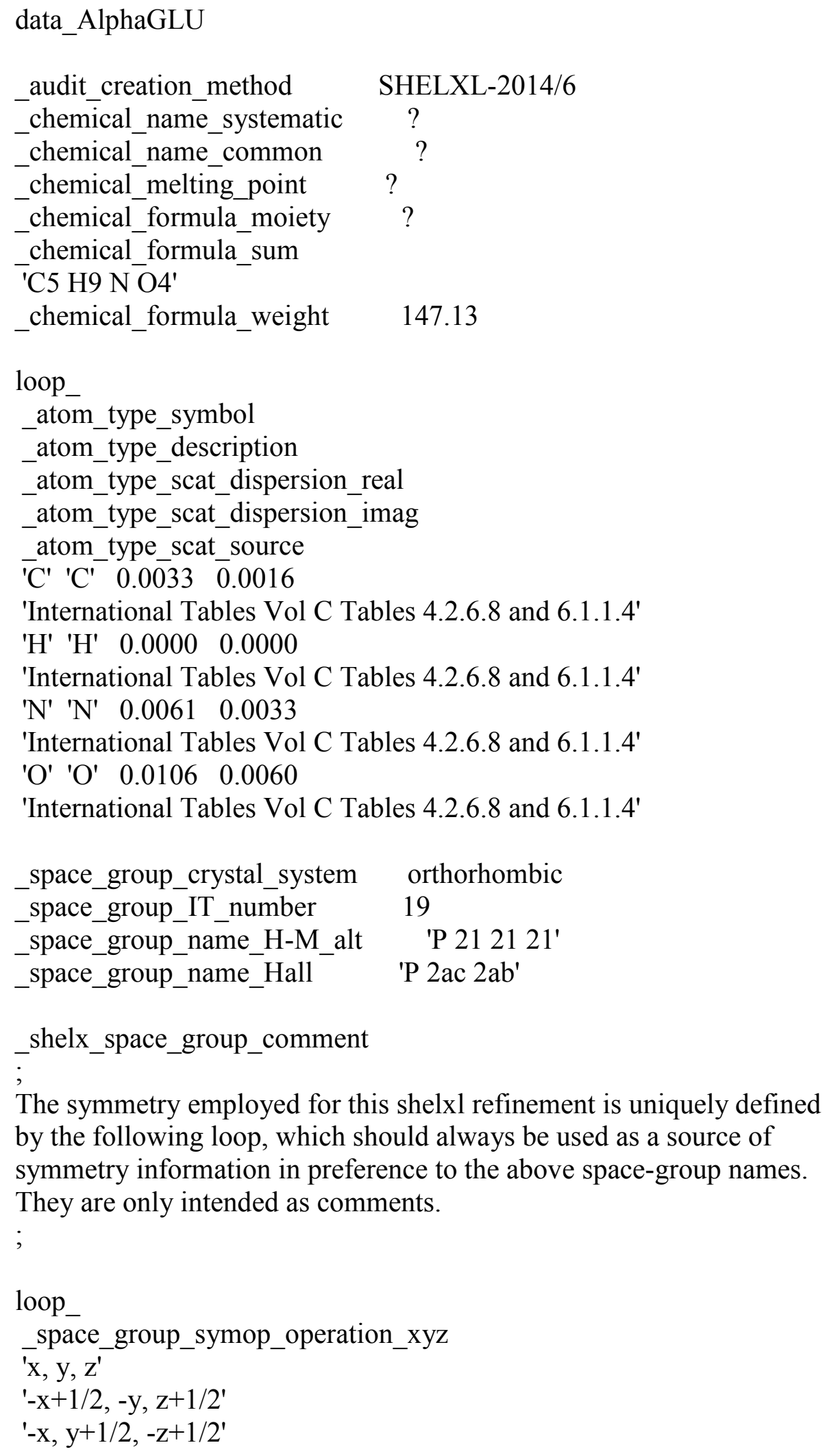




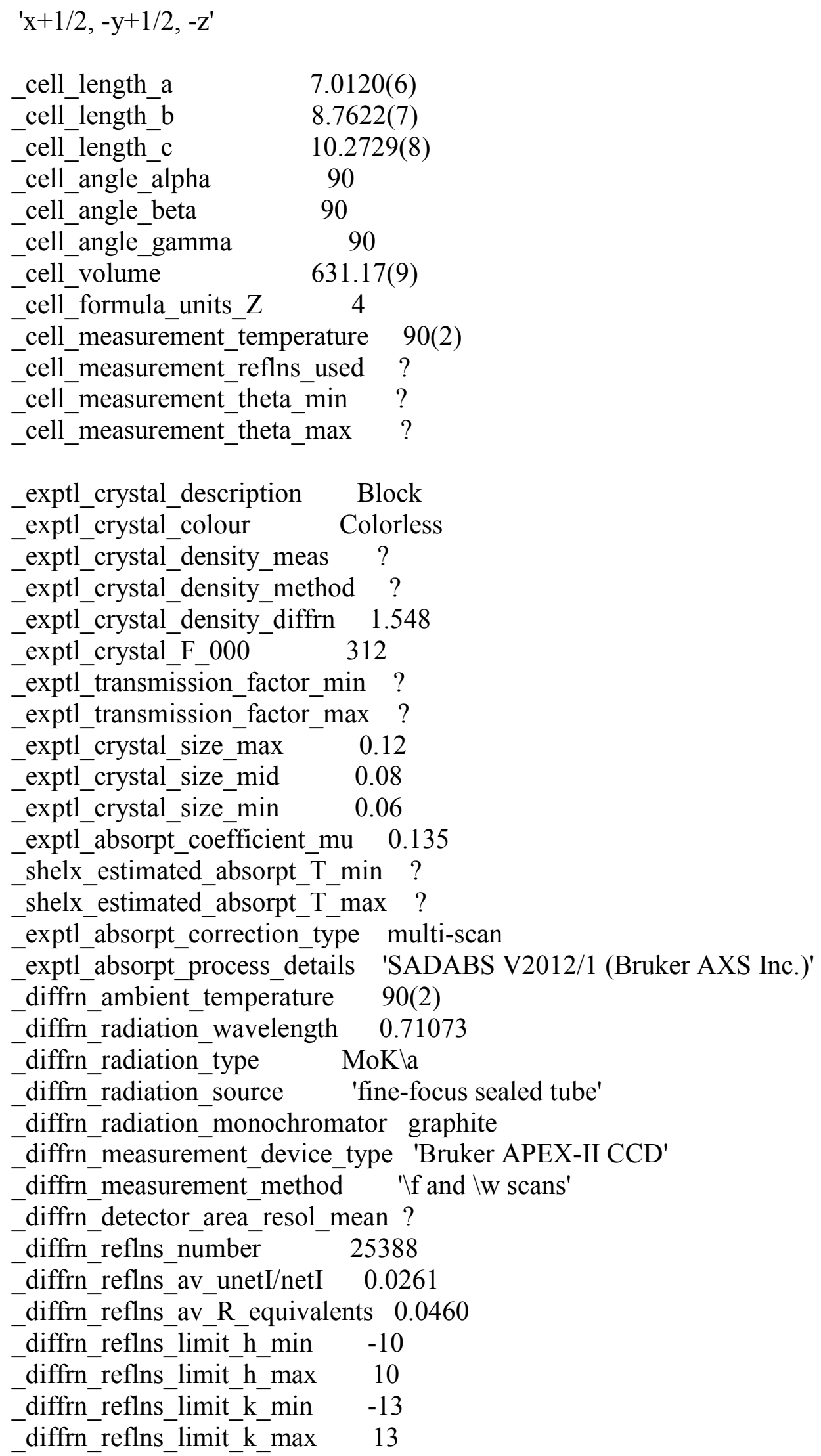




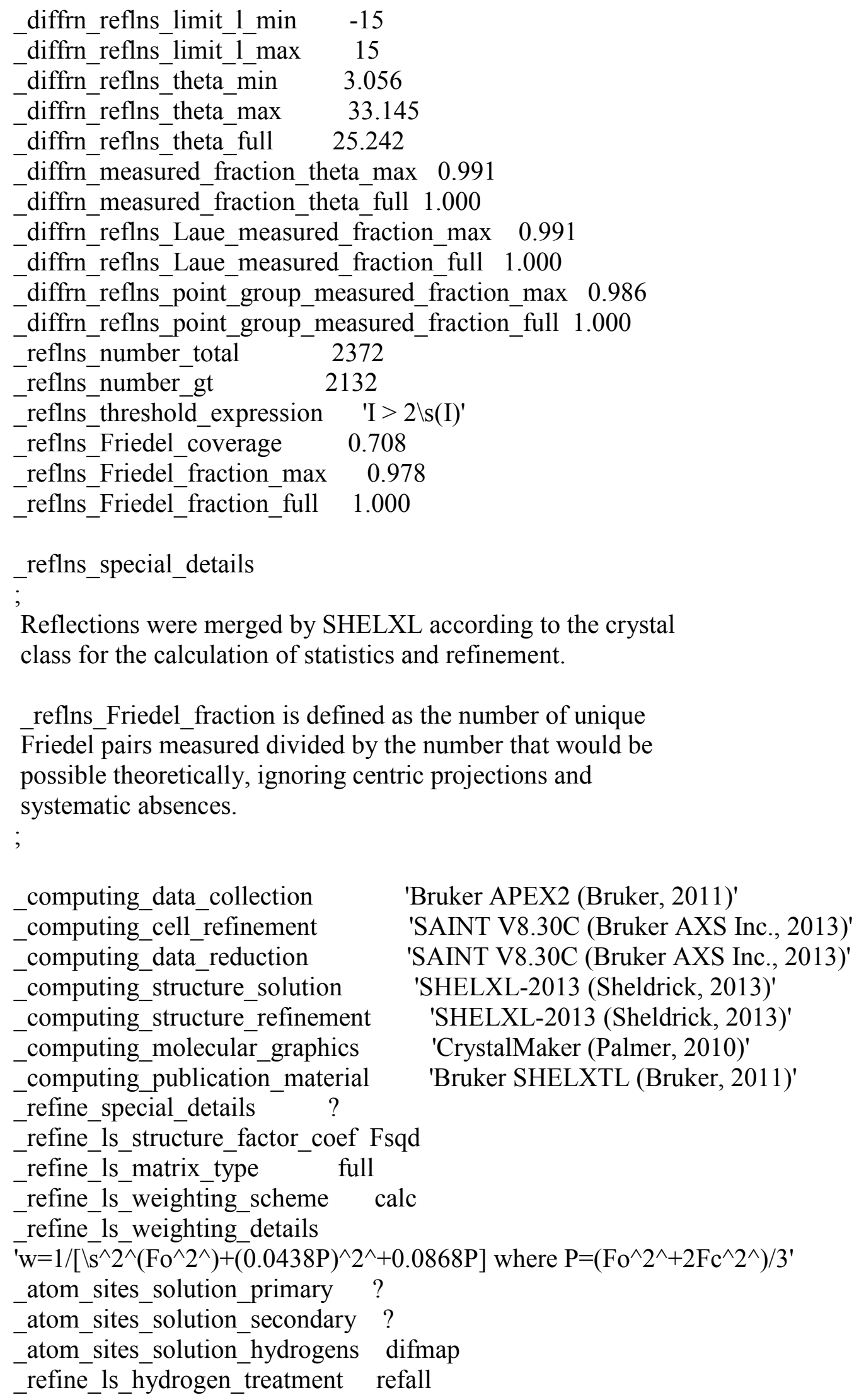


refine_1s_extinction_method none

refine_ls_extinction_coef.

refine_ls_abs_structure details

;

Flack $x$ determined using 835 quotients $[(\mathrm{I}+)-(\mathrm{I}-)] /[(\mathrm{I}+)+(\mathrm{I}-)]$

(Parsons, Flack and Wagner, Acta Cryst. B69 (2013) 249-259).

;

refine ls abs structure Flack -0.1(3)

chemical_absolute_configuration?

refine_ls_number_reflns 2372

_refine_ls_number_parameters 127

refine_ls_number_restraints 0

_refine_ls_R_factor_all 0.0431

_refine_ls_R_factor_gt $\quad 0.0335$

_refine_ls_wR_factor_ref 0.0788

_refine_ls_wR_factor_gt $\quad 0.0739$

refine ls goodness of fit ref 1.047

refine_ls_restrained_S_all 1.047

_refine_ls_shift/su_max 0.001

_refine_ls_shift/su_mean $\quad 0.000$

loop_

_atom_site_label

_atom_site_type_symbol

_atom_site_fract_x

_atom_site_fract_y

atom site fract $\mathrm{z}$

_atom_site_U_iso_or_equiv

_atom_site_adp_type

_atom_site_occupancy

_atom_site_site_symmetry_order

_atom_site_calc_flag

_atom_site_refinement_flags_posn

_atom_site_refinement_flags_adp

_atom_site_refinement_flags_occupancy

_atom_site_disorder_assembly

_atom_site_disorder_group

Ō001 O $0.8 \overline{7} 535(17) 0.59616(11) 0.58514(10) 0.0159$ (2) Uani $11 \mathrm{~d} \ldots .$.

O002 O 0.67471(15) 0.56552(12) 0.87814(9) 0.0145(2) Uani $11 \mathrm{~d} \ldots .$.

O003 O 0.70556(16) 0.76389(13) 1.01470(10) 0.0155(2) Uani $11 \mathrm{~d} \ldots . .$.

O004 O 0.88651(18) 0.81142(13) 0.46910(10) 0.0195(2) Uani $11 \mathrm{~d} \ldots .$.

N005 N 0.45804(17) 0.91948(14) 0.84805(11) 0.0118(2) Uani $11 \mathrm{~d}$. . . . .

C006 C 0.66795(18) 0.70685(15) 0.90638(12) 0.0109(2) Uani $11 \mathrm{~d}$. . . . .

C007 C 0.90141(19) 0.74462(15) 0.57311(13) 0.0123(2) Uani $11 \mathrm{~d}$. . . . .

C008 C 0.61017(18) 0.81631(15) 0.79670(12) 0.0102(2) Uani $11 \mathrm{~d}$. . . . .

C009 C 0.9485(2) 0.82369(17) 0.69938(13) 0.0139(2) Uani $11 \mathrm{~d} \ldots . .$. 
C00A C 0.7777(2) 0.91543(16) 0.75094(13) 0.0131(2) Uani $11 \mathrm{~d} \ldots .$.

H1 H 0.513(4) 0.987(3) 0.906(2) 0.030(6) Uiso $11 \mathrm{~d} \ldots .$.

H2 H 0.364(3) 0.861(2) 0.8862(18) 0.015(4) Uiso $11 \mathrm{~d} \ldots \ldots$

H3 H 0.405(3) 0.975(3) $0.778(2) 0.028(6)$ Uiso $11 \mathrm{~d} \ldots$.

$\mathrm{H} 4 \mathrm{H} 0.555(3) 0.763(2) 0.7248(19) 0.013(5)$ Uiso $11 \mathrm{~d} \ldots \ldots$

H5 H 0.846(4) 0.546(3) 0.510(2) 0.037(6) Uiso $11 \mathrm{~d} \ldots$.

H6 H 0.819(3) 0.975(2) 0.8222(19) 0.018(5) Uiso $11 \mathrm{~d} \ldots \ldots$

H7 H 0.734(3) 0.987(2) 0.681(2) 0.020(5) Uiso $11 \mathrm{~d} \ldots .$.

H8 H 0.989(3) $0.753(2) 0.764(2) 0.023(5)$ Uiso $11 \mathrm{~d} \ldots .$.

H9 H $1.053(3) 0.892(2) 0.679(2) 0.025$ (5) Uiso $11 \mathrm{~d} \ldots \ldots$ 


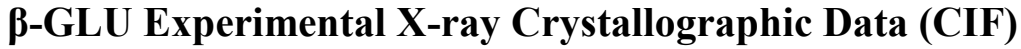

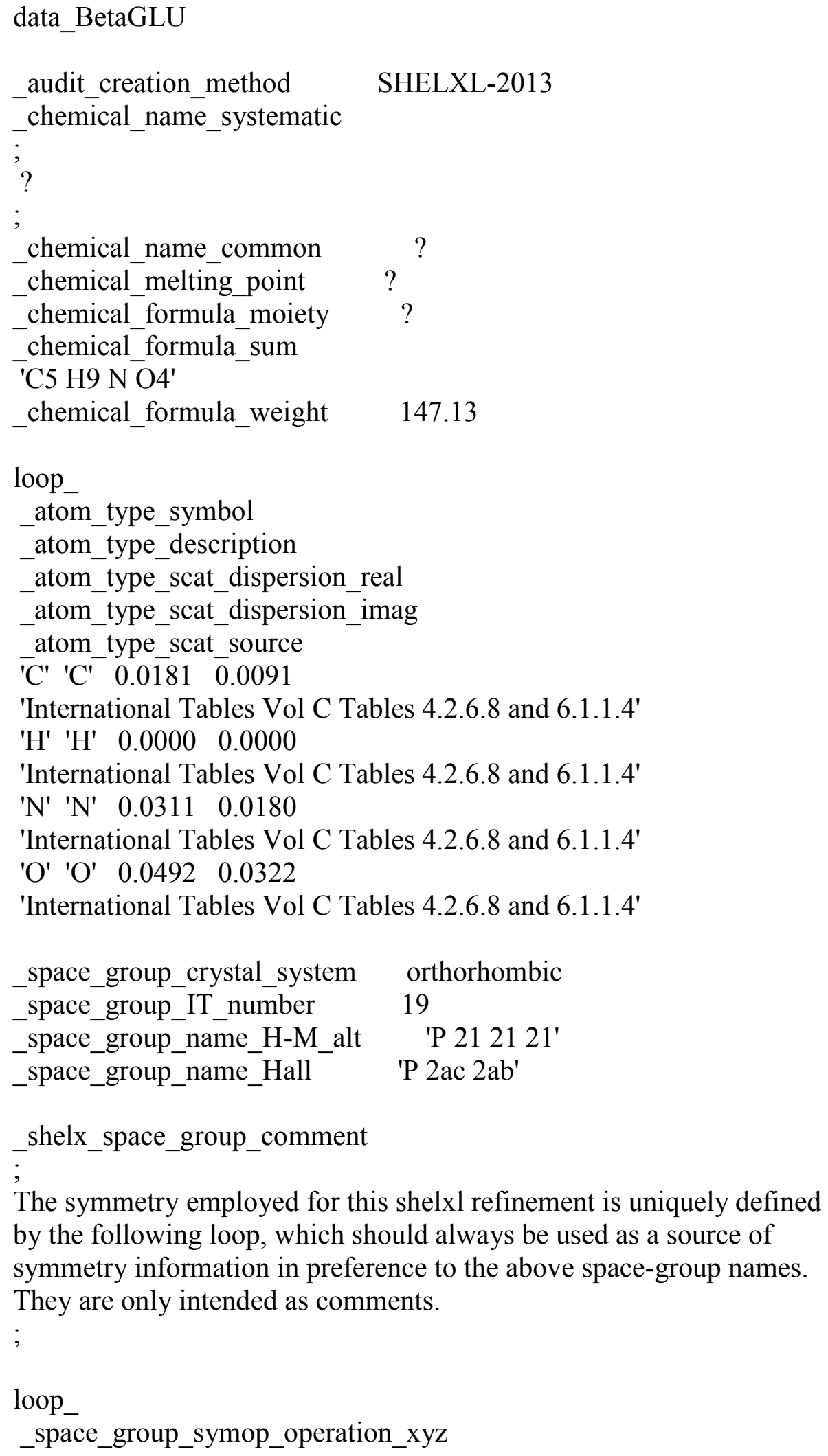




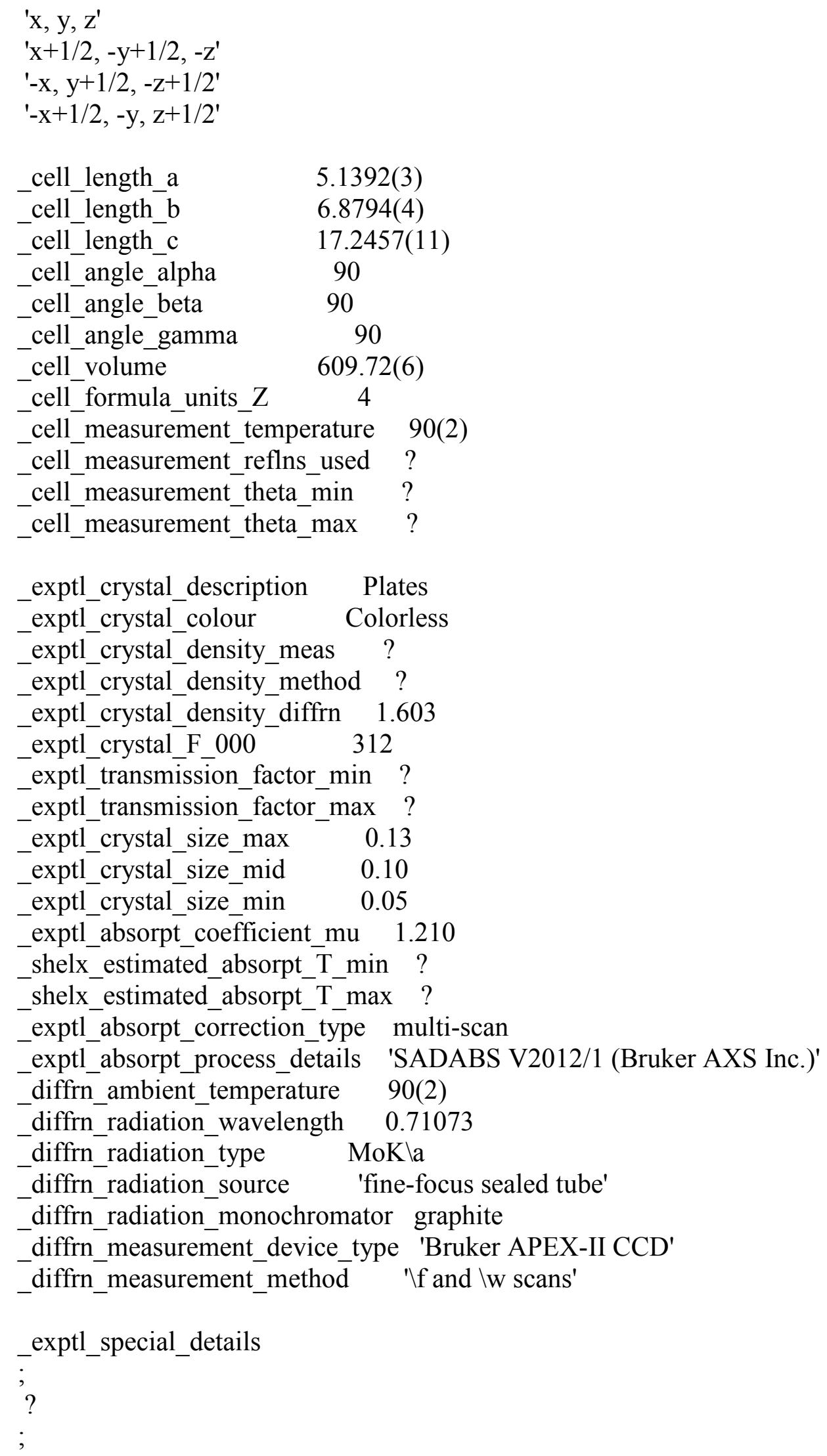




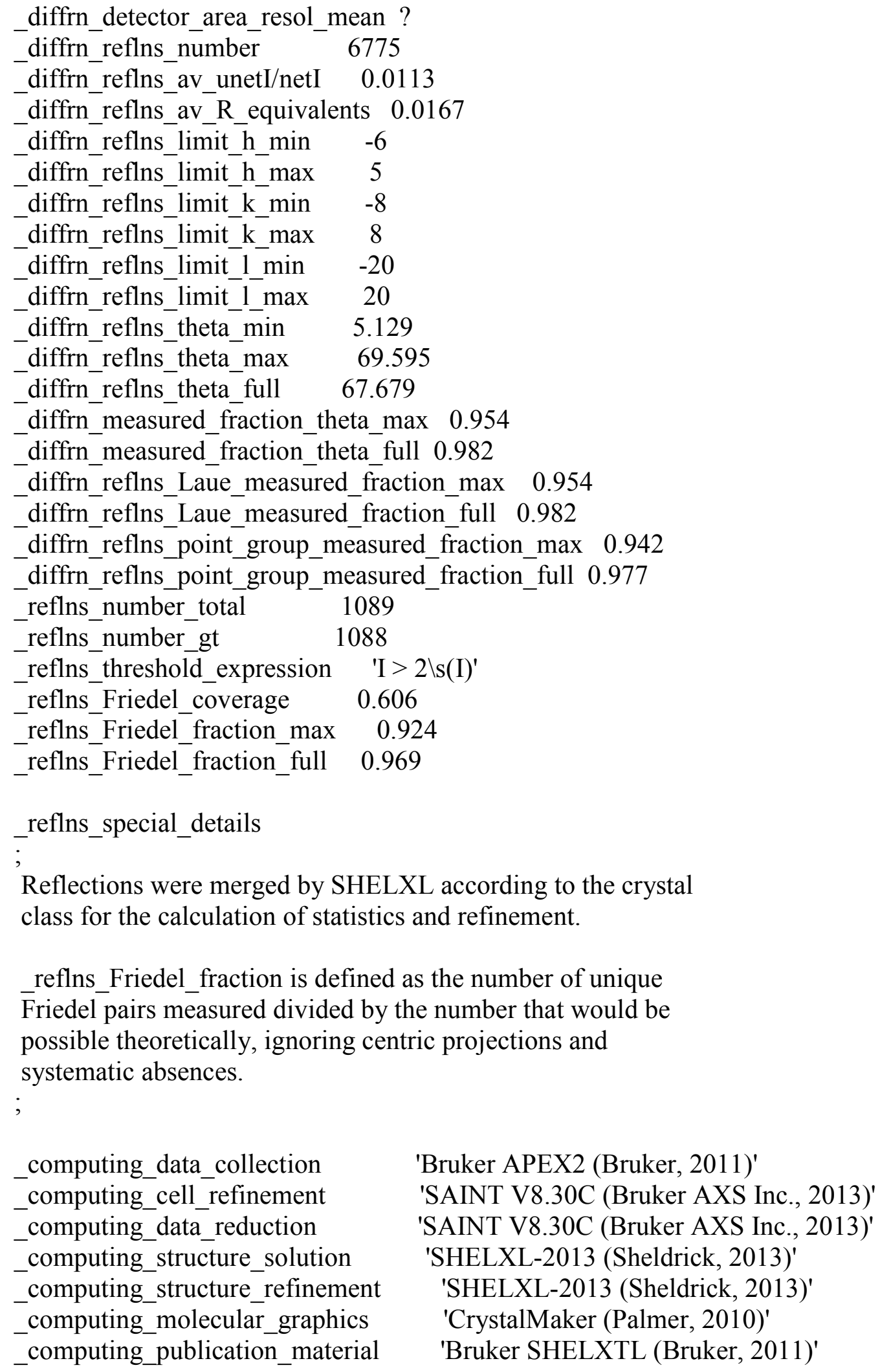

'Bruker APEX2 (Bruker, 2011)'

'SAINT V8.30C (Bruker AXS Inc., 2013)'

'SAINT V8.30C (Bruker AXS Inc., 2013)'

'SHELXL-2013 (Sheldrick, 2013)'

'SHELXL-2013 (Sheldrick, 2013)'

'CrystalMaker (Palmer, 2010)'

'Bruker SHELXTL (Bruker, 2011)' 


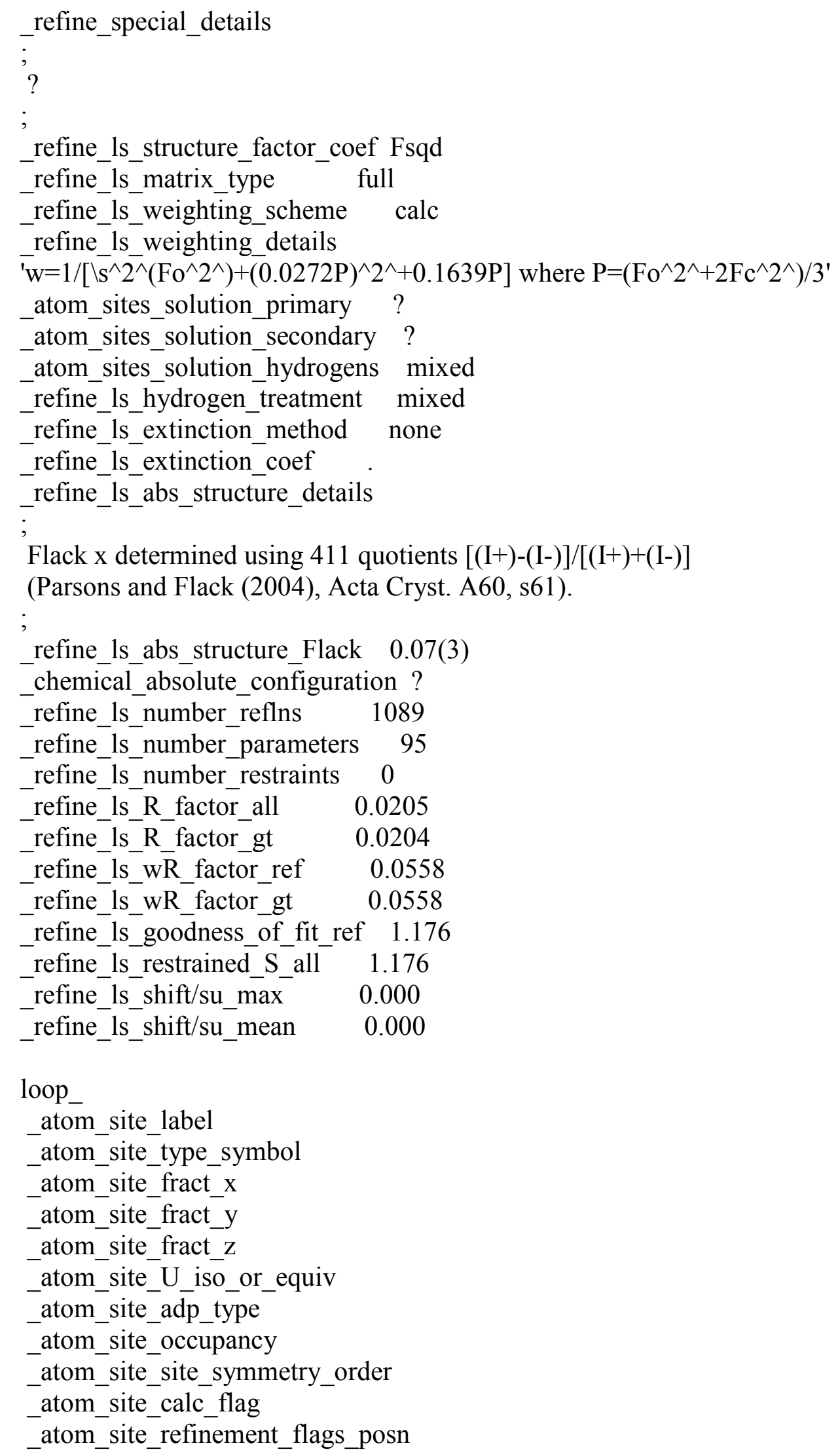




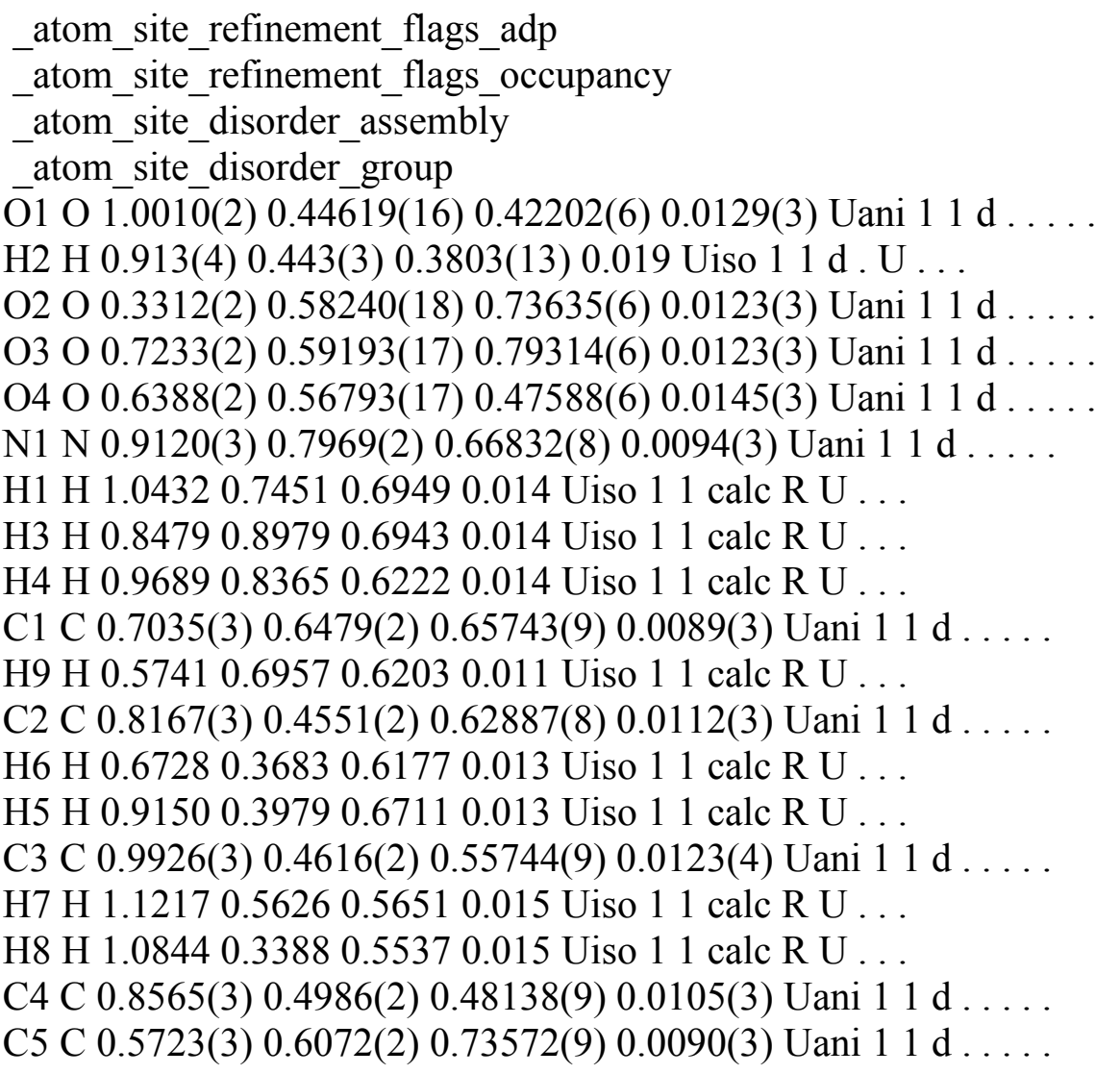

